\title{
Eccentricity Estimator for Wide-Angle Fovea Sensor by FMI Descriptor Approach
}

\author{
Sota SHIMIZU, Member, IEEE and J. W. BURDICK, Member, IEEE
}

\begin{abstract}
This paper proposes a method for estimating eccentricity that corresponds to an incident angle to a fovea sensor. The proposed method applies Fourier-Mellin Invariant descriptor for estimating rotation, scale, and translation, by taking both geometrical distortion and non-uniform resolution of a space-variant image by the fovea sensor into account. The following 2 points are focused in this paper. One is to use multi-resolution images computed by Discrete Wavelet Transform for reducing noise caused by foveation properly. Another is to use a variable window function (although the window function is generally used for reducing DFT leakage caused by both ends of a signal.) for changing an effective field of view (FOV) in order not to sacrifice high accuracy. The simulation compares the root mean square (RMS) of the foveation noise between uniform and non-uniform resolutions, when a resolution level and a FOV level are changed, respectively. Experimental results show that the proposed method is consistent with the wide-angle space-variant image by the fovea sensor, i.e., it does not sacrifice high accuracy in the central FOV.
\end{abstract}

\section{INTRODUCTION}

L OG-POLAR mapping, biologically-inspired by analytic formulation of cortical mapping of the primate visual system [1], is well-known as a method that does not only reduces image data size drastically but also gives rotationand scale-invariant (RS-invariant) property. In order to acquire the log-polar image, not only by software methods [2] but also by 2 kinds of hardware methods have been proposed and developed. One is a method by a special-made $\mathrm{CCD} / \mathrm{CMOS}$ chip, i.e., a retina-like sensor, where united size of photosensitive elements increases logarithmically as going to periphery [3]-[5]. The other is a method by special-made optical lens where projected image is highly distorted geometrically, i.e., a wide-angle foveated (WAF) lens [6]-[8]. In the latter case, the special-made lens is usually combined with a commercially-available Cartesian (i.e., linear-coordinate) vision chip, i.e., where the size of photosensitive elements is uniform (on the other hand, the former is combined with a commercially-available conventional lens). The authors have been doing research on fovea vision in terms of foveation modeling and its applications by using an actual WAF lens [9]-[11]. It is

Manuscript received February 15, 2008. This work was supported mainly by Wind \& Biomass Energy R\&D and Information Center

Sota Shimizu was with Division of Applied Science and Engineering, California Institute of Technology, Pasadena, CA 91125, USA (phone: +1-626-395-2359; e-mail: sato@caltech.edu) and is currently with Graduate School of Engineering Science, Osaka University, 1-3 Machikaneyama, Toyonaka, Osaka 560-8531, Japan.

Joel. W. Burdick is with Division of Applied Science and Engineering, California Institute of Technology, Pasadena, CA 91125, USA well-known that a conventional Cartesian vision sensor acquires an input image with uniform-resolution and gives it translation-invariant property. However, the resolution by the fovea sensors is not uniform in the entire field of view (FOV), it is the highest in the central FOV, and it decreases rapidly as going to periphery. That is, the input image by the fovea sensor has no longer a translation-invariant property in Discrete Fourier Transform (DFT), even if it is transformed into linear coordinates. On the other hand, as introduced in the opening sentence, the fovea sensor has an advantage that it can give us the log-polar image that is RS-invariant around the optical axis of the fovea sensor.

Figure 1(a) shows a linear-coordinate Target image $I$ of $512 \times 512$ [pixels]. Figures 1(b) and (c) are a distorted foveated image $D F$, computed from the image $I$ by Advanced Wide-Angle Foveated (AdWAF) model [8], and an undistorted foveated image $U D F$, remapped from the image $D F$ into a linear-coordinate space, in 3 cases of eccentricity, that corresponds to an incident angle to the sensor (both images have $128 \times 128$ [pixels] as their size). Watermark-like arias seen in the image $U D F$ of Fig.1(c) increases as the eccentricity gets larger. Such alias occurs when the image is up-sampled from low resolution to high resolution. Figure $1(\mathrm{~d})$ is the original log-polar image $O L P$ that is transformed from a part of the image $D F$ into polar coordinates. In order to keep the RS-invariant property mentioned above, we need to compensate deformation in the image OLP (this deformation increases highly as the eccentricity gets larger). Figure 1(e) is the compensated log-polar image CLP where the deformation is corrected from the image $O L P$ by the eccentricity compensator (EC) [9]. It is noted that the image quality decreases as the eccentricity gets larger.

A log-polar mapping of DFT magnitude of an input image is well-known as Fourier-Mellin Invariant (FMI) descriptor [12]-[14]. The input image by the fovea sensor is space-variant, originally. This means there are 2 factors, i.e., one is geometrical distortion of the image and the other is non-uniform resolution. Thus, we need to take the non-uniform resolution into account in order to apply FMI descriptor for the space-variant image, even if the geometrical distortion is removed. Section II of this paper proposes eccentricity estimator (EE) that estimates the eccentricity by applying FMI descriptor for multi-resolution images by Discrete Wavelet Transform. In addition, a variable window function is introduced in order not to sacrifice high accuracy in the central FOV. Section III examines the proposed method by a simulation of foveation noise from white Gaussian noise images and experiments. 


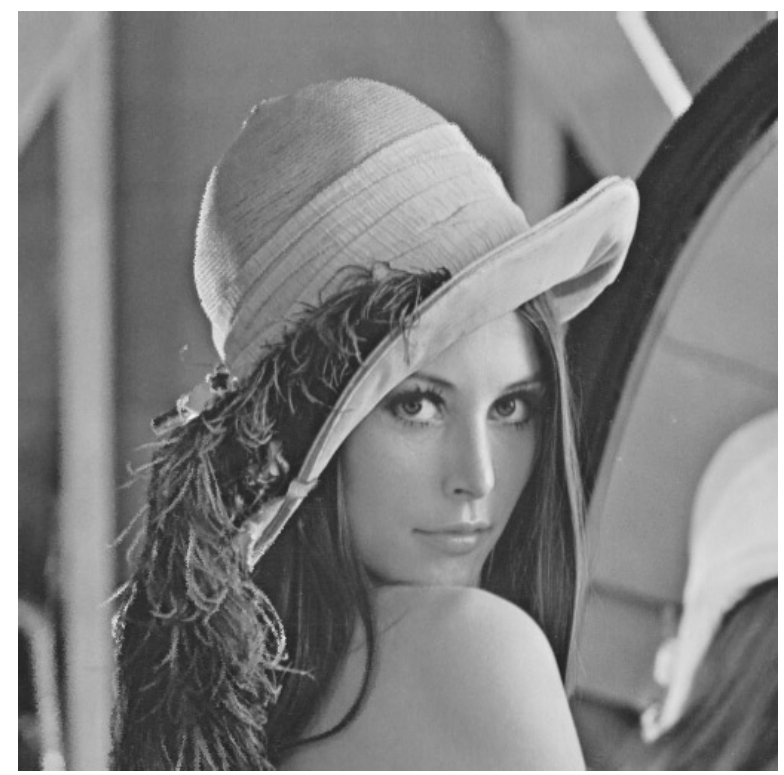

(a)Linear-coordainate target image (I) (of 512x512 [pixels])
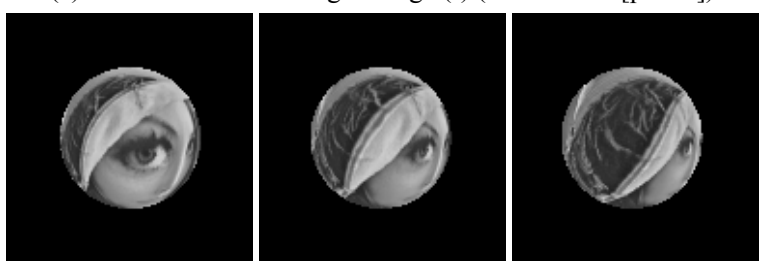

(b)distorted foveated image $(D F)$ (of 128x128)

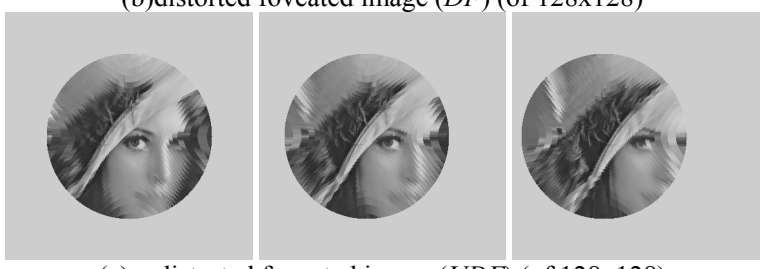

(c)undistorted foveated image $(U D F)$ (of 128x128)

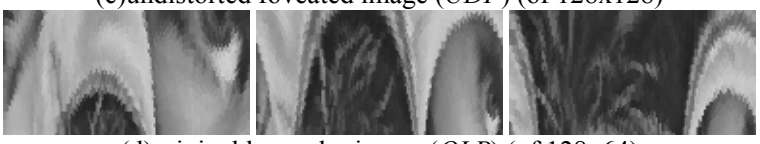

(d)original log-polar image $(O L P)$ (of 128x64)

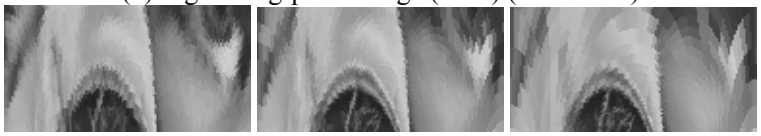

(e)compensated log-polar image (CLP) (of 128x64)

Fig. 1 Target image $I$, distorted foveated image $(D F)$, undistorted foveated image $(U D F)$, original log-polar image $(O L P)$ and compensated log-polar image $(C L P)$ in each eccentricity $\theta_{\varepsilon}\left(=0,18.69\right.$ and $34.08\left[^{\circ}\right]$ from the left $)$

\section{ECCENTRICITY ESTIMATOR}

\section{A. Linear-coordinate Image with Space-variant Resolution}

As shown in Fig. 1(b), an input image acquired from the fovea sensor is distorted highly because the image height from the image center (corresponding to its optical axis) is not linear to the object height. This paper applies Advanced Wide Angle Foveated (AdWAF) model [8] for a concrete foveation model of the fovea sensor. The AdWAF model combines planar and spherical projections with linear coordinates and logarithmic coordinates, respectively. Thus, the FOV is divided into 4 regions: fovea $\left(0 \leq \theta \leq \theta_{0}\right)$, para-fovea ( $\left.\theta_{0} \leq \theta \leq \theta_{1}\right)$, near-periphery $\left(\theta_{1} \leq \theta \leq \theta_{2}\right)$, and periphery $\left(\theta_{2} \leq \theta \leq \theta_{\max }\right)$. Figure 2 compares an AdWAF image and a linear-coordinate image by pinhole camera (PHC) lens model. In this simulation, image intensity is changed in order to make it easy to distinguish boundaries of the FOV, i.e., $\theta_{0}=9.826\left[^{\circ}\right], \theta_{1}=19.107\left[^{\circ}\right], \theta_{2}=34.715\left[^{\circ}\right]$, and $\theta_{\max }=60.0\left[^{\circ}\right]$. The AdWAF model and the PHC lens model are defined in the following.

\section{AdWAF model:}

$$
r=\left\{\begin{array}{ll}
r_{\max } c_{0} f_{1} \tan \theta & \left(0 \leq \theta \leq \theta_{0}\right) \\
r_{\max }\left\{c_{1} \log _{a} f_{1} \tan \theta+d_{1}\right\} & \left(\theta_{0} \leq \theta \leq \theta_{1}\right) \\
r_{\max }\left\{c_{2} \log _{b}\left(f_{2} \theta\right)+d_{2}\right\} & \left(\theta_{1} \leq \theta \leq \theta_{2}\right) \\
r_{\max }\left\{c_{3} f_{2} \theta+d_{3}\right\} & \left(\theta_{2} \leq \theta \leq \theta_{\max }\right)
\end{array},\right.
$$

where $f_{1}$ and $f_{2}$ are focal lengths for planar projection and spherical projection, basis $a$ and basis $b$ are given by $a=\exp \left(1 / f_{1} \tan \theta_{0}\right)$ and by $b=\exp \left(1 / f_{2} \theta_{2}\right)$, respectively.

The $r_{\max }$ is the maximum image height when $\theta=\theta_{\max }, c_{i}(i=0$, $1,2,3)$ is a scale modification factor for adjusting image height partly in each region, and $d_{i}(i=1,2,3)$ is determined from continuity of the image height and its magnification.

\section{PHC lens model:}

$$
r=L \tan \theta \quad\left(0 \leq \theta \leq \theta_{\text {max }}\right) .
$$

where $L$ is a distance from the optical center $O_{c}$ to the object plane,

$$
L=\frac{r_{\max }}{\tan \theta_{\max }} .
$$

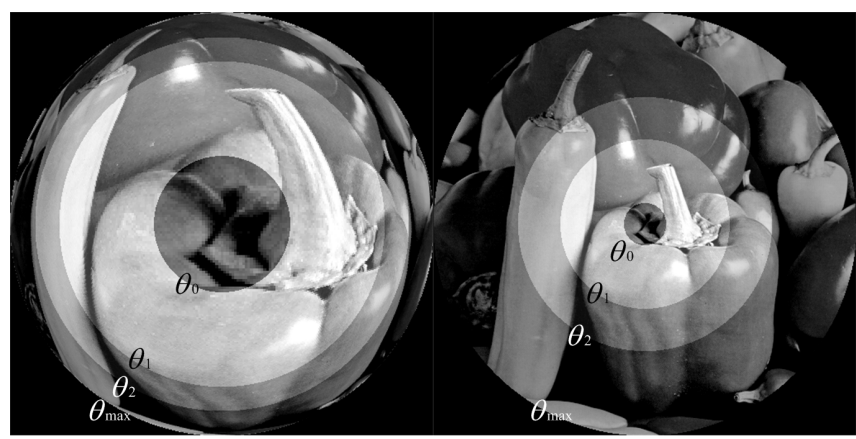

(a)AdWAF image

(b)linear-coordinate image

Fig. 2 Comparison of AdWAF image and linear-coordinate image

Assume that a target image $I$ is in the direction of eccentricity $\theta_{\varepsilon}$ and its rotation $\phi_{\varepsilon}$ on the object plane. That is, the eccentricity is an incident angle to the fovea sensor, as shown in Fig.3.

The incident direction, $(\theta, \phi)$, from a point $(x, y)$ on the image $I$ to the optical center of the fovea sensor is expressed,

$$
\left\{\begin{array}{l}
\theta=\tan ^{-1}\left\{\sqrt{\left(x+\varepsilon \cos \phi_{\varepsilon}\right)^{2}+\left(y+\varepsilon \sin \phi_{\varepsilon}\right)^{2}} / L\right\}, \\
\phi=\tan ^{-1}\left\{\left(y+\varepsilon \sin \phi_{\varepsilon}\right) /\left(x+\varepsilon \cos \phi_{\varepsilon}\right)\right\}
\end{array}\right.
$$

where $\varepsilon$ is positional eccentricity on the object plane.

$$
\varepsilon=L \tan \theta_{\varepsilon} .
$$




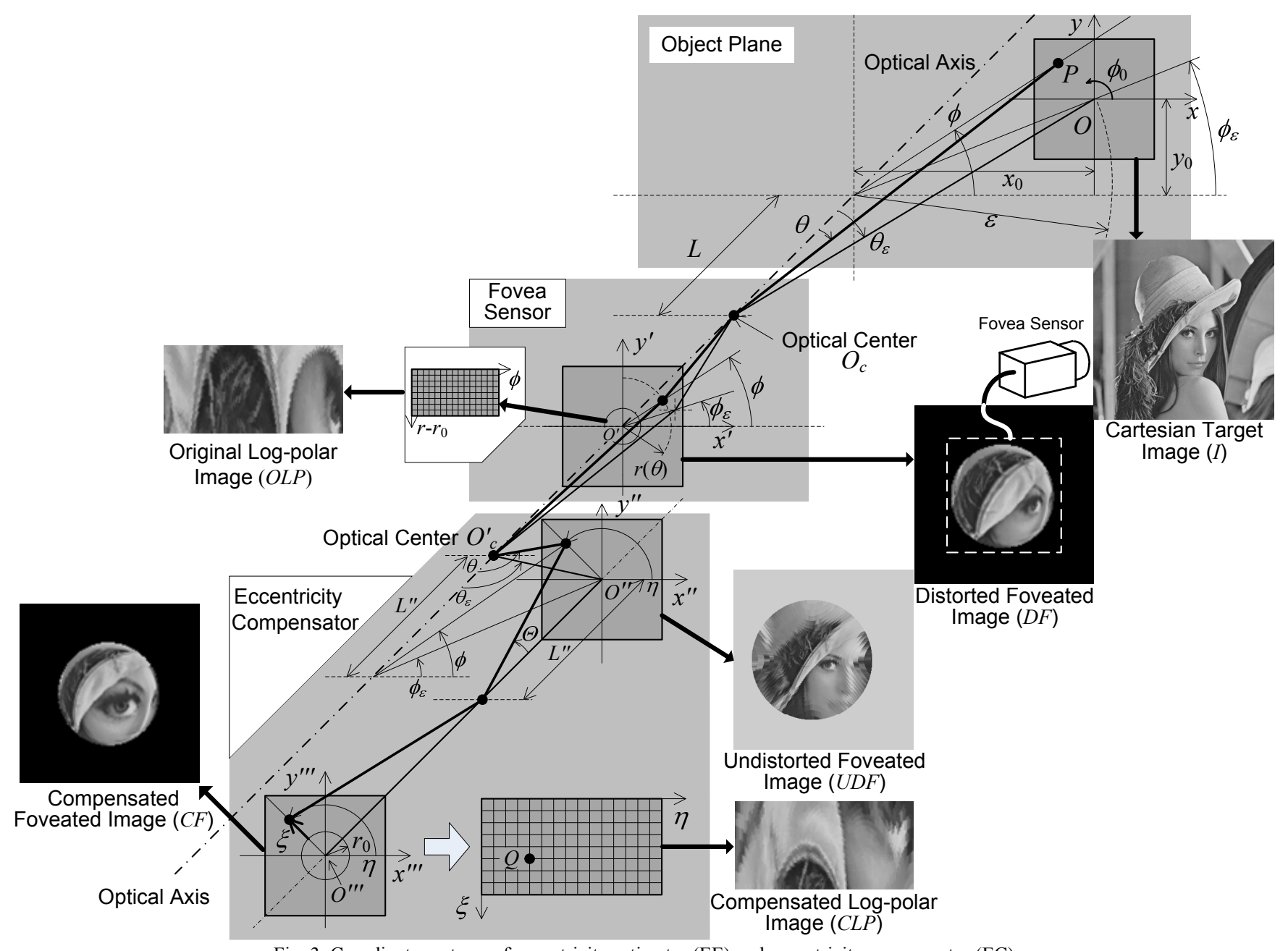

Fig. 3 Coordinate systems of eccentricity estimator (EE) and eccentricity compensator (EC)

Coordinates $\left(x^{\prime}, y^{\prime}\right)$, of a distorted foveated image $D F$ that corresponds to the input image by the fovea sensor, are given by eq. (6).

$$
\left\{\begin{array}{l}
x^{\prime}=\alpha_{1} r(\theta) \cos \phi \\
y^{\prime}=\alpha_{1} r(\theta) \sin \phi
\end{array},\right.
$$

where $r(\theta)$ is the image height of the fovea sensor in terns of incident angle $\theta$, and $\alpha_{1}$ is magnification of the image $D F$.

Coordinates $\left(x^{\prime}, y^{\prime}\right)$, of an undistorted foveated image $U D F$ with linear-coordinates, are expressed in eq. (7).

$$
\left\{\begin{array}{l}
x^{\prime \prime}=\alpha_{2}\left(L \tan \theta \cos \phi-\varepsilon \cos \phi_{\varepsilon}\right) \\
y^{\prime \prime}=\alpha_{2}\left(L \tan \theta \sin \phi-\varepsilon \sin \phi_{\varepsilon}\right)
\end{array},\right.
$$

where $\alpha_{2}$ is magnification of the image $U D F$.

Both the image $D F$ and the image $U D F$ have space-variant resolution. Both images are mapped using mappings $f_{D F}$ and $f_{U D F}$ of an image warping technique, respectively.

$$
\begin{aligned}
& f_{D F}: I \rightarrow D F, \\
& f_{U D F}: D F \rightarrow U D F,
\end{aligned}
$$

\section{B. Fourier-Mellin Invariant descriptor}

Figure 4(a) shows the image $f$ and the image $g$ transformed from the $f$ with rotation $\phi_{0}[\mathrm{rad}]$, scale $\mu$, translation $\left(x_{0}, y_{0}\right)$.

$$
\begin{array}{r}
g(x, y)=f\left(\mu\left(x \cos \phi_{0}+y \sin \phi_{0}\right)-x_{0},\right. \\
\left.\mu\left(-x \sin \phi_{0}+y \cos \phi_{0}\right)-y_{0}\right)
\end{array} .
$$

Fourier-Mellin Invariant (FMI) descriptor based on Fourier Mellin Transform is well-known for extracting rotation-, scale-, and translation-invariant feature [12]-[14]. FMI descriptor is equivalent with a log-polar mapping of DFT magnitude of the input image, around the DC component. This paper refers to this method for realistic implementation [13][14] in order to compute rotation, scale, and translation between the 2 images. FMI descriptor is derived simply in the following.

Denote DFTs of the image $f$ and the image $g$ as $F(m, n)$ and $G(m, n)$, and their log-polar mappings as $f_{l p}$ and $g_{l p}$.

$$
g_{l p}(\phi, \lambda)=\mu^{-2} f_{l p}\left(\phi-\phi_{0}, \lambda-\sigma\right) \quad,
$$

where $\lambda=\log \sqrt{m^{2}+n^{2}}$ and $\sigma=\log (\mu)$. Note that the log-polar mapping transforms rotation and scale to translation. DFTs of the log-polar mappings $f_{l p}$ and $g_{l p}$ are expressed as 


$$
G_{l p}(v, \varpi)=\mu^{-2} e^{-j 2 \pi\left(v \sigma+\varpi \phi_{0}\right)} F_{l p}(v, \varpi) .
$$

This equation means translation $\left(\phi_{0}, \sigma\right)$ can be determined from the $f_{l p}$ and $g_{l p}$ using phase correlation method [13][15]. Rotation and scale are computed from this translation.

Now, another image $f^{\prime}$ is mapped from the image $f$ using computed rotation $\phi_{0}$ and scale $\mu$.

$$
\begin{array}{r}
f^{\prime}(x, y)=f\left(\mu\left(x \cos \phi_{0}+y \sin \phi_{0}\right),\right. \\
\left.\mu\left(-x \sin \phi_{0}+y \cos \phi_{0}\right)\right)
\end{array}
$$

Translation $\left(x_{0}, y_{0}\right)$ between the image $g$ and the image $f^{\prime}$ is computed using the phase correlation method, again.

$$
\frac{G(n, m) F^{\prime^{*}}(n, m)}{\left|G(n, m) F^{\prime}(n, m)\right|}=e^{j 2 \pi\left(n x_{0}+m y_{0}\right)} .
$$

Eccentricity and its rotation are denoted as,

$$
\left\{\begin{array}{l}
\theta_{\varepsilon}=\tan ^{-1}(\varepsilon / L) \\
\phi_{\varepsilon}=\tan ^{-1}\left(y_{0} / x_{0}\right)
\end{array},\right.
$$

where $\varepsilon=\sqrt{x_{0}^{2}+y_{0}^{2}}$.

When FMI descriptor is applied for the images $f, f$, and $g$, a window function is used. In addition, the following high-pass filter is used for DFTs $F$ and $G$ [13].

$$
\begin{aligned}
H P F(n, m) & =(1.0-\cos (\pi n) \cos (\pi m)), \\
& *(2.0-\cos (\pi n) \cos (\pi m))
\end{aligned}
$$

where $-0.5 \leq n, m \leq 0.5$.
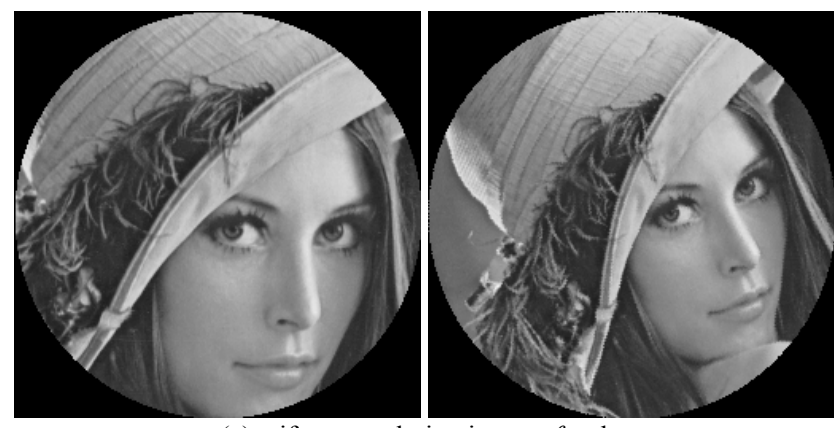

(a)uniform-resolution images $f$ and $g$

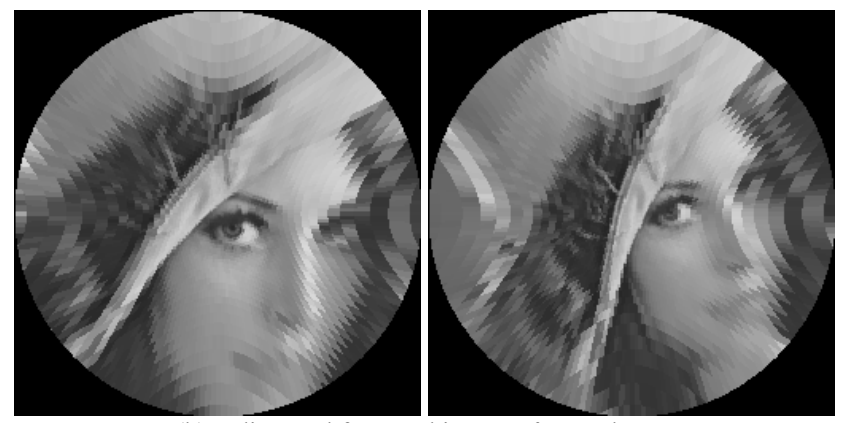

(b)undistorted foveated images $f_{U D F}$ and $g_{U D F}$

Fig.4 An image and a transformed image

using rotation, scale and translation

\section{Applying to Space-variant Image UDF}

FMI descriptor assumes to be applied for a linear-coordinate (Cartesian) image. However, even if the image $D F$ by the fovea sensor is transformed into the linear-coordinate image $U D F$ by removing distortion, we need to take non-uniform resolution into account. Multi-resolution images $f_{j}$ and $g_{j}$ (where $j$ is resolution level), computed from the images $f$ and $g$ by using Discrete Wavelet Transform (DWT), are shown in Fig.5. Haar wavelet is used for this computation as a mother wavelet. The proposed method applies FMI descriptor for the multi-resolution image from low level to high level, and estimates eccentricity on a reliable level $j_{m}$ using phase correlation peak as criterion. In addition, the proposed method also defines a variable window function with FOV level $\lambda$ in order not to sacrifice high accuracy in the central FOV. That is, the higher-level eccentricity is estimated by changing an effective FOV smaller, based on the eccentricity estimated when the window size is the largest. This paper applies Hanning window for the variable window function.

\section{Hanning window:}

$$
\left\{\begin{aligned}
w n d(x, y) & =0\left(\sqrt{x^{2}+y^{2}}>L \tan \theta_{\max } \cdot l^{\lambda}\right) \\
& \left.=0.5-0.5 \cos \left(2 \pi \sqrt{x^{2}+y^{2}}\right)\right) \\
& \left(0 \leq \sqrt{x^{2}+y^{2}} \leq L \tan \theta_{\max } \cdot l^{\lambda}\right)
\end{aligned}\right.
$$

Figure 6 shows the image $f$ after applying the window function with different FOV level $\lambda$. Figure 7 is a block diagram of the proposed method.

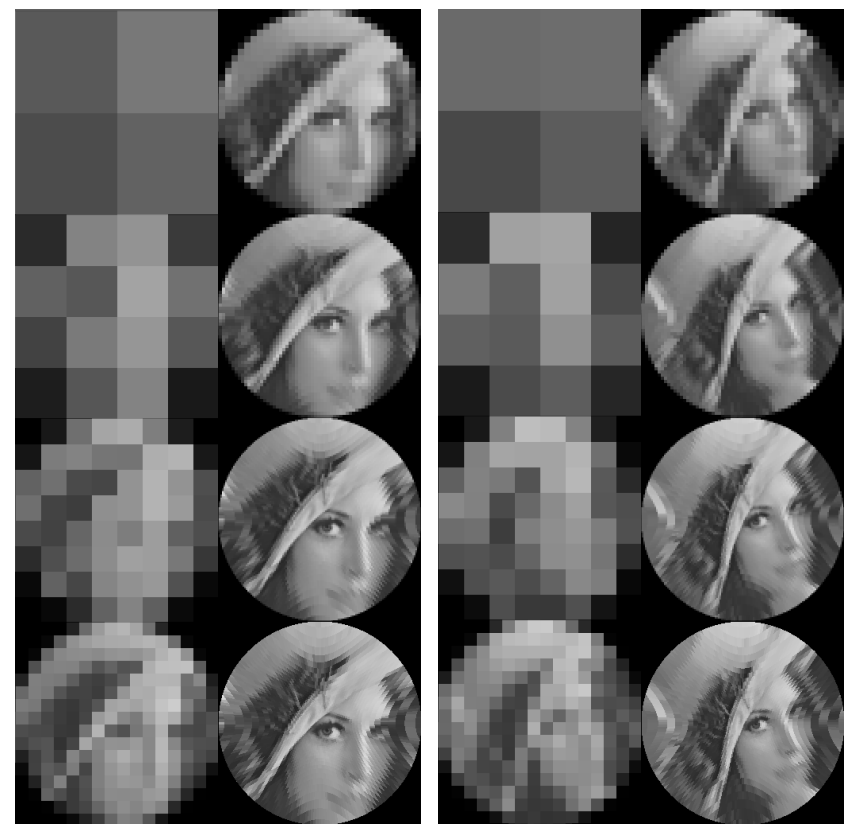

Fig.5 Multi-resolution images $f_{j}, g_{j}$ by Haar wavelet

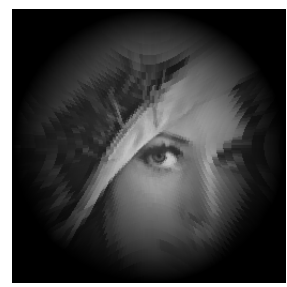

(a) $\lambda=0$

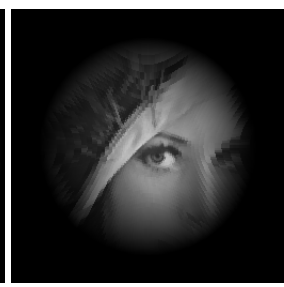

(b) $\lambda=1$

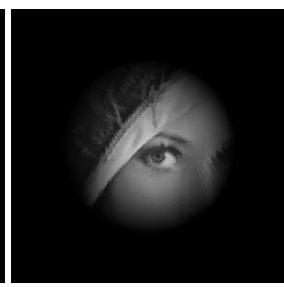

(c) $\lambda=2$
Fig. 6 Image $f$ when using different size of a window function 


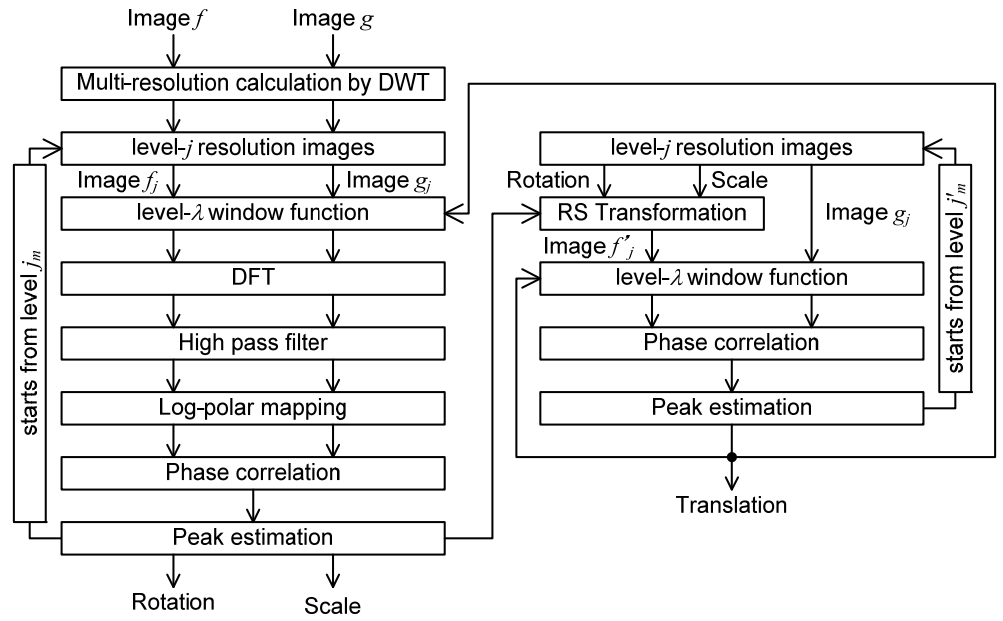

Fig. 7 Block diagram of the proposed method
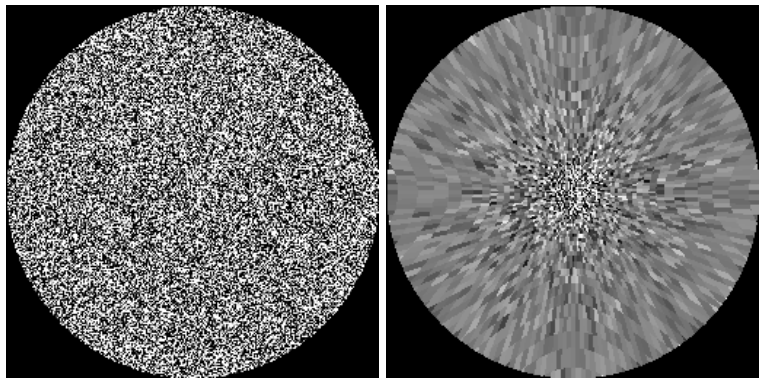

(a)a white Gaussian noise (WGN) image $w$ and its $U D F$-image $w_{U D F}$

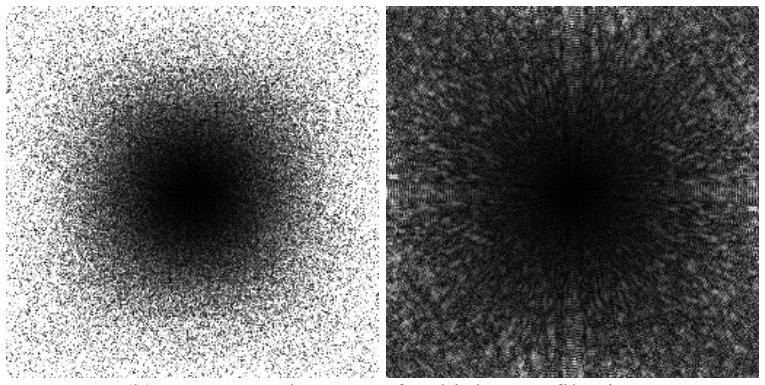

(b)DFTs $W$ and $W_{U D F}$, after high-pass filtering

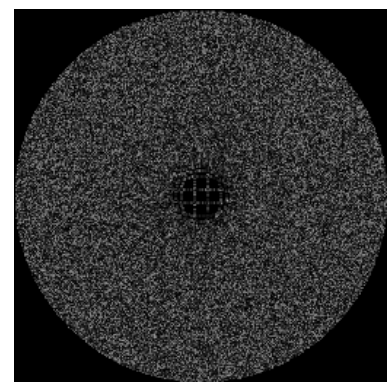

(c)Foveation noise image $w_{f n}$

Fig. 8 Comparison of a WGN image $w$ and its $U D F$ image $w_{U D F}$

\section{EXAMINATION}

\section{A. Foveation Noise}

The proposed method uses high frequency components emphasized by high-pass filter for the phase correlation method. However, it is obvious that many of high frequency components in the image of Fig.4(a) are lost in the corresponding image $U D F$ of Fig.4(b). This loss causes a risk of wrong estimation by the phase correlation. Thus, in order to reduce this risk, the proposed method estimates the eccentricity by applying FMI descriptor for the multi-resolution image from a lower-level image where resolution is less space-variant.

Figure 8(a) compares a white Gaussian noise image $w$ and its $U D F$ image $w_{U D F}$. Figure $8(\mathrm{~b})$ shows that magnitude of their DFTs $W$ and $W_{U D F}$ (where the high-pass filter is applied) is influenced by foveation mainly in high frequencies. Figure $8(\mathrm{c})$ is a foveation noise image $w_{f n}$ that shows absolute value of difference between Invert DFTs of $W$ and $W_{U D F}$. Root Mean Square $(R M S)$ of image intensity is used for estimating the foveation noise.

$$
R M S=\sqrt{\frac{\left\|w_{f n}\right\|^{2}}{N_{x} \cdot N_{y}}},
$$

where $N_{x} \mathrm{x} N_{y}$ is an image size of the image $w_{f n}$.

Figure 9 shows the resolution level versus average of $R M S$ of the foveation noise image computed from 100 white Gaussian noise images in each level. This simulation uses AdWAF model in conditions of $\theta_{0}=9.826\left[^{\circ}\right], \theta_{1}=19.107\left[^{\circ}\right]$, $\theta_{2}=34.715\left[^{\circ}\right]$, and $\theta_{\max }=60.0\left[^{\circ}\right]$ for foveation. In addition, 3 cases of the variable window function with a different FOV level (where a basis $l$ is 0.7 ) are compared with a case without the window function. Consistency between the proposed method and properties of wide-angle fovea sensor (i.e., wide-angle FOV and high resolution in the central FOV) are examined as follows.

1) $R M S$ of the foveation noise is zero when the resolution level $j$ is less than 4 , and increases as the level $j$ gets larger. This indicates that the resolution level should be decreased as the eccentricity is estimated in a wider-angle FOV.

2) As the FOV level $\lambda$ of the variable window function gets larger (i.e., the FOV gets narrower), RMS gets smaller. This indicates that the FOV level should be increased as the eccentricity is estimated in the higher resolution. 


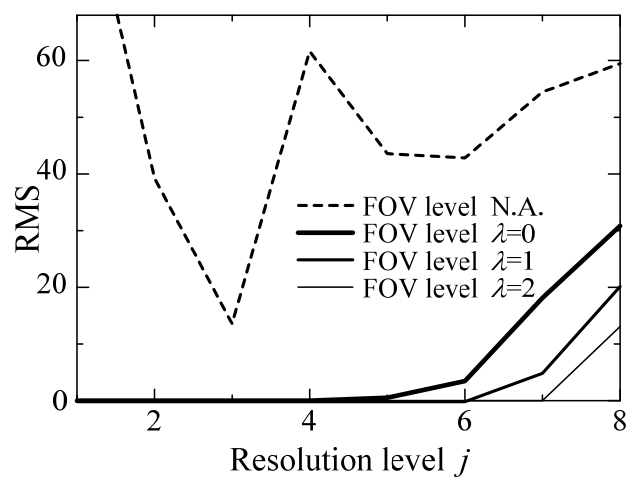

Fig.9 RMS of foveation noise image

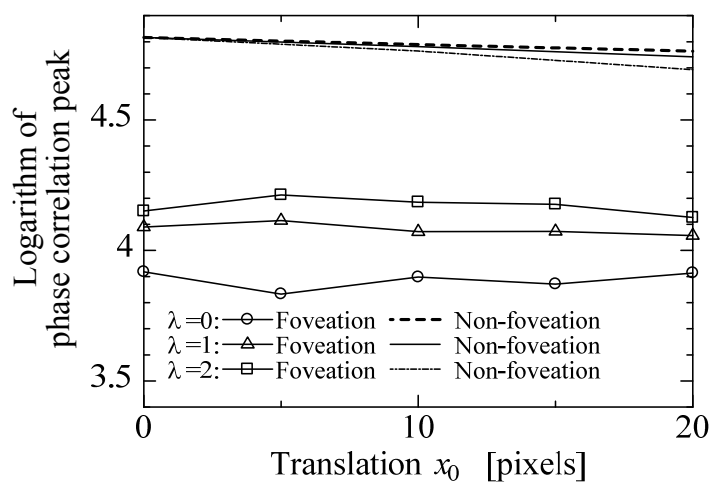

(a) when $\mu=0.8$

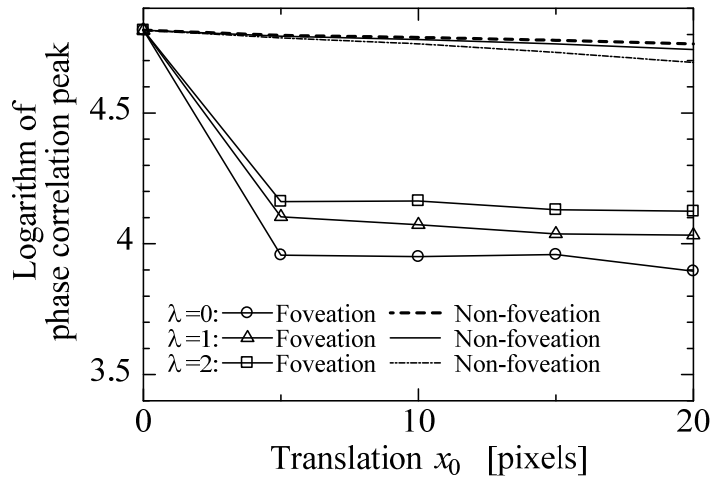

(b) when $\mu=1.0$

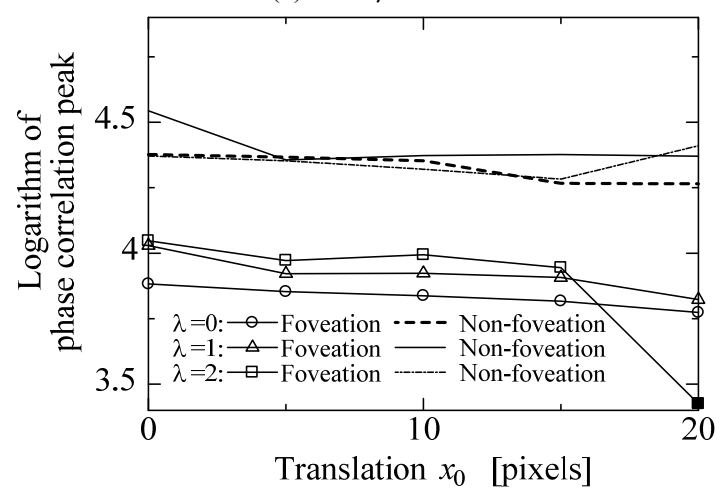

(c) when $\mu=1.2$

Fig.10 Experimental results

\section{B. Experiments}

Figure 10 shows experimental results. Being given the translation $x_{0}$, logarithms of phase correlation peak determined automatically by the proposed estimation method are compared between the uniform-resolution Lenna image and its $U D F$ image, in 3 cases of the scale $\mu=0.8,1.0$, and 1.2 (where $y_{0}=0$ and $\phi_{0}=0\left[^{\circ}\right]$ ). In these results, all cases except when $\mu=1.2$ and $x_{0}=20$ (shown as a black square) have succeeded in estimating the translation $x_{0}$ by an error less than 1 [pixel] (Also, the scale and rotation are estimated accurately). The wrong estimation case mentioned above occurs when the highest FOV level $\lambda=2$ (i.e., the narrowest FOV). A local maximal value seems to be selected wrongly because the foveation noise reduces the determined phase correlation peak largely. We note that the higher FOV level gives us the higher value of the phase correlation peak. This means that the high resolution in the central FOV can be preserved by the variable window function, although the high FOV level has a weakness of the translation.

\section{ACKNOWLEDGMENT}

The authors thank to Dr. Hao Jiang, California Institute of Technology, for his many helps.

\section{REFERENCES}

[1] Schwartz, E.L., Spatial mapping in the primate sensory projection: Analytic structure and relevance to perception, Biological Cybernetics, Vol.29 (1977), pp.181-194.

[2] Sandini, G. and Tagliasco, V., An anthropomorphic retina-like structure for scene analysis, Computer Graphics and Image Processing, 14 (1980), pp.365-372.

[3] Berton, F., Sandini, G. and Metta, G., Anthropomorphic Visual Sensors, Encyclopedia of Sensors, Edited by C.A. Grimes, E.C.Dickey and M.V.Pishko, Vol. 10 (2005), pp. 1-16.

[4] Van der Spiegel, J., Kreider, G., Claeys, C., Debusschere, I., Sandini, G., Dario, P. and et al., A foveated retina-like sensor using CCD technology, Analog VLSI Implementations of Neural Networks. Kluwer, C.Mead and M.Ismail, Boston (1989).

[5] Wodnicki, R., W.Roberts, G. and Levine, M.D., A foveated image sensor in standard CMOS technology, In Custom Integrated Circuits Conference, Santa Clara, California (1995).

[6] Shimizu, S. et.al., Vision Sensor with Wide Angle and High Distortion lens, Video proceedings of IEEE International Conference on Robotics and Automation (1995), Visual Sensing 3.

[7] Kuniyoshi, Y., Kita, N., Sugimoto, K., et.al., A Foveated Wide Angle Lens for Active Vision, Proc. of IEEE International Conference on Robotics and Automation (1995), pp.2982-2988.

[8] Shimizu, S., Wide-Angle Foveation for All-Purpose Use, IEEE/ASME transaction on Mechatronics (published).

[9] Shimizu, S. and Burdick, J., Eccentricity Compensator for Log-Polar Sensor, 2007 IEEE International Conference on Robotics and Automation (2007), pp.4214-4219

[10] Shimizu, S., Multi-Functional Application of Wide Angle Foveated Vision Sensor in Mobile Robot Navigation, Journal of Robotics and Mechatronics, vol.14, No.4 (2002), pp.382-389.

[11] Shimizu, S., Wide-Angle Vision Sensor with High-Distortion Lens (Detection of Camera Location and Gaze Direction Based on the Two-Parallel-Line Algorithm), JSME International Journal, Series C, Vol.41, No.4 (1998), pp.893-900.

[12] Casasent, D. and Psaltis, D., Position, Rotation and Scale-invariant Optical Correlation, Applied Optics, Vol. 15 (1976.), pp.1795-1799.

[13] Reddy, B.S. and Chatterji, B.N., An FFT-based Technique for Translation, Rotation and Scale-Invariant Image Registration, IEEE Transactions on Image Processing, Vol.5, No.8 (1996), pp.1266-1271.

[14] Chen, Q., Defrise, M. and Deconinck, F., Symmetric Phase-Only Matched Filtering of Fourier-Mellin Transforms for Image Registration and Recognition, IEEE Transaction on Pattern Analysis and Machine Intelligence, Vol. 16, No.12 (1994), pp. 1156-1168.

[15] Horner, J. and Gianino, D., Phase-only matched filtering, Applied Optics, Vol.23, No.6 (1984), pp. 812-816. 